\title{
CONTROLLABILITY AND OBSERVABILITY OF LINEAR DELAY SYSTEMS: AN ALGEBRAIC APPROACH
}

\author{
M. FLIESS AND H. MOUNIER
}

\begin{abstract}
Interpretations of most existing controllability and observability notions for linear delay systems are given. Module theoretic characterizations are presented. This setting enables a clear and precise comparison of the various examined notions. A new notion of controllability is introduced, which is called $\pi$-freeness.
\end{abstract}

\section{INTRODUCTION}

Although the linear systems with delays may be among the simplest classes of infinite dimensional systems, many concurrent extensions of Kalman's controllability and observability have been proposed in the literature. These definitions stem either from a functional analytic approach (see, e.g., $[1],[2],[3],[30],[48]$ ) or from a more algebraic viewpoint (see, e.g., [5], [7], $[23],[45])$.

Our aim is here twofold: firstly, to classify and compare most of the existing structural properties; secondly, to introduce a new controllability concept, which seems to be quite relevant in many concrete situations.

We work in a module theoretic framework, rather different from the one introduced by Kalman [22], and which directly extends previous works on finite dimensional systems $[10,11,13,14]$. A linear delay system is then a finitely generated module $\Lambda$ over the ground ring $k\left[\frac{d}{d t}, \delta\right]$, where $k$ is a commutative field and $\delta=\left(\delta_{1}, \ldots, \delta_{r}\right)$ are the delay operators.

In this setting, a typical result (see Section 6 for further details) is the interpretation of the important notion of reachability (the latter being developed by [45], [5], among others):

Proposition 1.18. Let $\Lambda$ be the $k\left[\frac{d}{d t}, \delta\right]$-module generated by $x_{1}, \ldots, x_{n}$, $u_{1}, \ldots, u_{m}$ subject to the equations

$$
\dot{\boldsymbol{x}}=F(\boldsymbol{\delta}) \boldsymbol{x}+G(\boldsymbol{\delta}) \boldsymbol{u}
$$

M. Fliess: Centre de Mathématiques et Leurs Applications, École Normale Supérieure de Cachan, 61, avenue du Président Wilson, 94235 Cachan, France. E-mail: fliess@cmla.ens-cachan.fr \& fliess@lss.supelec.fr.

H. Mounier: Département AXIS, Institut d'Électronique Fondamentale, Bâtiment 220, Université Paris-Sud, 91405 Orsay Cedex, France. E-mail: mounier@ief.u-psud.fr.

This work was partially supported by the European Commission's Training and Mobility of Researchers (TMR) Contract \# ERBFMRX-CT970137, by the G.D.R. Medicis and by the G.D.R.-P.R.C. Automatique.

Received by the journal June 23, 1997. Revised March 16, 1998. Accepted for publication August 31, 1998.

(C) Société de Mathématiques Appliquées et Industrielles. Typeset by IATEX. 
where $\boldsymbol{x}=\left(x_{1}, \ldots, x_{n}\right), \boldsymbol{u}=\left(u_{1}, \ldots, u_{m}\right), F(\boldsymbol{\delta}) \in k[\boldsymbol{\delta}]^{n \times n}$ and $G(\boldsymbol{\delta}) \in$ $k[\delta]^{n \times m}$. Then, $(\star)$ is reachable if and only if $\Lambda$ is free.

This result is a direct consequence of the resolution, due to Quillen [40] and Suslin [47], of Serre's famous conjecture [44]. Recall that in the finite dimensional case where the system module is over $k\left[\frac{d}{d t}\right]$ (i.e., over a principal ideal domain), Kalman's controllability amounts to the freeness (or torsion freeness) of this module [10]. The equivalence between torsion freeness and freeness for modules is no longer valid over $k\left[\frac{d}{d t}, \boldsymbol{\delta}\right]$ (see [42]).

Weak controllability, which is considered by [31], [39], [28], among others, is shown to be equivalent to

$$
\operatorname{rk}_{k(\boldsymbol{\delta})}\left[A(\boldsymbol{\delta}), A(\boldsymbol{\delta}) B(\boldsymbol{\delta}), \ldots, A(\boldsymbol{\delta})^{n-1} B(\boldsymbol{\delta})\right]=n
$$

where $k\left(\frac{d}{d t}, \delta\right)$ is the quotient field of $k\left[\frac{d}{d t}, \delta\right]$. The module associated to such a weakly controllable system, considered over $k(\delta)\left[\frac{d}{d t}\right]$ through an extension of the ground ring, is free. This leads us to another technique, namely varying the rings under consideration through suitable tensor products, in the spirit of what is done along Grothendieck's ideas in modern algebraic geometry (see, e.g., [20], [21]). This key ingredient in obtaining the various comparisons also gives rise to a new form of controllability. Let the $k\left[\frac{d}{d t}, \delta\right]$ module $\Lambda$ be torsion free. Then, there exists an element $\pi$ in $k[\delta]$ such that $k\left[\frac{d}{d t}, \delta, \pi^{-1}\right] \Theta_{k[d / d t, \boldsymbol{\delta}]} \Lambda$ is free. The system will be called $\pi$-free. In many concrete examples (see, e.g. $[32,17,35]), \pi$ may be chosen as a single delay $\delta_{i}$.

The paper is organized as follows. We begin with general definitions and results for systems associated with modules over a general algebra containing $k\left[\frac{d}{d t}, \boldsymbol{\delta}\right]$. We then state some criteria for checking freeness and torsion freeness which are related to Quillen-Suslin's result. This is exploited in Section 4 for extending some coprimeness notions to delay systems. Section 5 demonstrates that many systems often encountered in practice and exhibiting a transmission delay are $\pi$-free. Section 6 interprets the notions of reachability and weak controllability. There, we employ a paper by one of the authors [33], where some of the commonly used functional analytic controllabilities are interpreted in an algebraic setting. A comparison diagram is given in Section 7 summarizing the previous comparison results. The final section is devoted to similar results on observability.

Most of the commutative algebra we use may be found in standard textbooks, such as $[9,27,42]$. In particular, the basic definitions of module, ideal, free module, ..., can be found for example in pages 11 to 17 of [9]. The notion of torsion freeness is in $[27$, p. 388] or $[42$, p. 224]. For projectivity, see, e.g., [9, A3.2 p. 615] or [42, Th. 3.11 and 3.14, p. 62-63]. For more elaborate results, we give the precise reference.

Our results were announced in $[16,17]$ and fully developed in [32].

A companion paper [35] deals with an example of $\pi$-free system, a vibrating string with an interior mass. Other examples may be found in $[32,36]$ and an application to feedback stabilization in [34]. 


\section{2. $R$-SYSTEMS}

\subsection{Preliminary definitions}

All the rings and algebras are commutative, with unity, and without zero divisors.

Definition 2.1. Let $R$ be a ring. An $R$-linear system, or an $R$-system, or a (linear) system over $R, \Sigma$ is a finitely generated $R$-module.

In other words, an $R$-system is an $R$-module with finite free presentation (see, e.g., [9, p.17], [42, p. 90]):

$$
E \stackrel{\phi}{\longrightarrow} W \longrightarrow \Sigma \longrightarrow 0
$$

A presentation matrix $P_{\Sigma}$ of $\Sigma$ is one associated with $\phi$.

Notation. We denote by $[\xi]$ the $R$-submodule spanned by a subset $\xi$ of $\Sigma$.

Definition 2.2. An $R$-linear dynamics, or an $R$-dynamics, or a (linear) $d y$ namics over $R$, is an $R$-system $\Sigma$ equipped with an input $\boldsymbol{u}=\left(u_{1}, \ldots, u_{m}\right)$, i.e., a finite subset of $\Sigma$, such that the quotient module $\Sigma /[\boldsymbol{u}]$ is torsion. The input $\boldsymbol{u}$ is said to be independent if $[\boldsymbol{u}]$ is a free $R$-module of rank $m$.

DeFinition 2.3. An output $\boldsymbol{y}=\left(y_{1}, \ldots, y_{p}\right)$ is a finite subset of $\Sigma$. An $R$-system equipped with an input and an output is called an input-output $R$-system.

\subsection{Controllability and observability}

Definition 2.4. Let $A$ be an $R$-algebra. The $R$-system $\Sigma$ is said to be $A-$ torsion free controllable (resp. A-projective controllable, A-free controllable) if the $A$-module $A \otimes_{R} \Sigma$ is torsion free (resp. projective, free).

Elementary homological algebra [42] yields:

Proposition 2.5. The A-free controllability (resp. the A-projective controllability) implies the A-projective controllability (resp. the A-torsion free controllability).

Definition 2.6. An $R$-system $\Sigma$ with input $\boldsymbol{u}$ and output $\boldsymbol{y}$ is said to be $A$-observable if the two $A$-modules $A \otimes_{R} \Sigma$ and $A \otimes_{R}[\boldsymbol{u}, \boldsymbol{y}]$ coincide.

\section{3. $\pi$-FREENESS}

The following result is borrowed from [43, Proposition 2.12.17, p. 233]:

Theorem and Definition 2.7. Let $\Sigma$ be an $R$-system, $A$ an $R$-algebra, and $\mathcal{S}$ a multiplicative part of $A$ such that $\Sigma$ is $\mathcal{S}^{-1} R$-free controllable. Then, there exists an element $\pi$ in $\mathcal{S}$ such that $\Sigma$ is $R\left[\pi^{-1}\right]$-free controllable. The preceding system will then be called $\pi$-free.

We have the following direct consequence:

Corollary 2.8. Let $\Sigma$ be an $R$-torsion free controllable $R$-system and $\mathcal{S}$ a multiplicative part of $R$ such that $\mathcal{S}^{-1} R$ is a principal ideal ring. Then, there exists $\pi \in \mathcal{S}$ such that $\Sigma$ is $R\left[\pi^{-1}\right]$-free controllable and $\Sigma$ is $\pi$-free. 


\subsection{EXamples Where $R$ is a PRincipal ideal Ring}

Take for $R$ the principal ideal ring $k[\xi]$ of polynomials in one indeterminate $\xi$ over a commutative field $k$. This case encompasses finite-dimensional continuous time systems $[10]$ if $\xi=\frac{d}{d t}$, finite-dimensional discrete time systems [12] if $\xi$ is a shift operator, as well as, for instance, systems with fractional derivatives if $\xi$ stands for some fractional derivation operator [15]. Well-known properties of finitely generated modules over principal ideal rings (see, e.g., [27, Chapter 3 , Section 7]) tell us that $k[\xi]$-projective (resp. $k[\xi]$-torsion free) controllable systems a re $k[\xi]$-free controllable. We will say, for short, that such a $k[\xi]$-system is controllable (see [10] for the connection with more classic approaches). A $k[\xi]$-system $\Lambda$ with input $\boldsymbol{u}$ and output $\boldsymbol{y}$ is said to be observable if, and only if, the two $k[\xi]-\operatorname{modules} \Lambda$ and $[\boldsymbol{u}, \boldsymbol{y}]$ coincide (see [10] for the connection with more classic approaches). See $[4,11,13,14]$ for several fruitful consequences of this framework.

\subsection{The CASE OF DELAY Systems}

Let $R$ be the ring $k\left[\xi_{0}, \xi_{1}, \ldots, \xi_{r}\right]$ of polynomials in $r+1$ indeterminates $\xi_{0}, \xi_{1}, \ldots, \xi_{r}$ over a commutative field $k$. Here, $\xi_{0}=\frac{d}{d t}, \xi_{i}=\delta_{i}, i=1, \ldots, r$, where $\delta_{i}$ is a (localized) delay operator ${ }^{1}$, we are thus dealing with (localized) delay systems. Then, $k\left[\xi_{0}, \xi_{1}, \ldots, \xi_{r+1}\right]$-torsion free controllability, $k\left[\xi_{0}, \xi_{1}, \ldots, \xi_{r+1}\right]$-projective controllability, and $k\left[\xi_{0}, \xi_{1}, \ldots, \xi_{r+1}\right]$-free controllability are no longer equivalent. Nevertheless we have, as a direct consequence of Corollary 2.8:

Proposition 2.9. A $k\left[\frac{d}{d t}, \boldsymbol{\delta}\right]$-torsion free controllable $k\left[\frac{d}{d t}, \boldsymbol{\delta}\right]$-system $\Lambda$ is $\pi$-free, where $\pi$ may be chosen in $k[\delta]$.

REMARK 2.10. In the case of a single delay, it is quite simple to obtain the element $\pi$ of the preceding proposition. Let $\Im_{\Lambda}$ be the ideal of $k\left[\frac{d}{d t}, \delta\right]$ generated by the maximal order minors of a presentation matrix of $\Lambda\left(\Im_{\Lambda}\right.$ is the Fitting ideal of $\Lambda$ ) and let $\widetilde{I}_{\Lambda}$ be the ideal of $k[s, z]$ obtained by substituting $s$ to $d / d t$ and $z$ to $\delta$.

Let $\left(s_{0}^{i}, z_{0}^{i}\right)$ be the common zeros of the generators of $\widetilde{I}_{\Lambda}$, and let $\widetilde{I}_{\Lambda}^{i}$ be the ideal of $\bar{k}[z]$ obtained by fixing $s$ to $s_{0}^{i}$. Let then $\pi_{i}$ be the lcd of the generators of $\widetilde{\mathfrak{I}}_{\Lambda}^{i}$. In the decomposition over $\bar{k}[z]$ in prime factors of $\pi_{i}$ :

$$
\pi_{i}=\prod_{j=1}^{N_{i}} \sigma_{j}^{\alpha_{j}}
$$

the various $\sigma_{j}$ are first degree polynomials of $\bar{k}[z]$. Denote by $\widetilde{\sigma}_{j}(\delta)$ the polynomial of $\bar{k}[z]$ corresponding to $\sigma_{j}$. It suffices to take

$$
\pi(\delta)=\prod_{i} \prod_{j=1}^{N_{i}} \tilde{\sigma}_{j}(\delta)
$$

\footnotetext{
${ }^{1}$ For a function $f: \mathbb{R} \rightarrow \mathbb{R}$, a (localized) delay operator $\delta_{i}$ is defined by $\delta_{i} f(t)=f\left(t-h_{i}\right)$, where $h_{i} \in \mathbb{R}_{+}$is a constant. The vector space spanned by the $h_{i}$ 's over the field $\mathbb{Q}$ of rational numbers is assumed to be $r$-dimensional. 
REMARK 2.11. Notice that noncommutative rings appear naturally in the study of non-stationary delay systems. The ground field $k$ above can then be taken as a differential-difference field (see [8]), and $k\left[\frac{d}{d t}, \delta_{1}, \ldots, \delta_{r}\right]$ is noncommutative.

From now on, $k$ will designate a commutative field with characteristic zero, such as $\mathbb{R}$ or $\mathbb{C}$, and a $k\left[\frac{d}{d t}, \boldsymbol{\delta}\right]$-system will be called a delay system.

\section{CRITERIA FOR $k\left[\frac{d}{d t}, \boldsymbol{\delta}\right]$-FREE AND $k\left[\frac{d}{d t}, \boldsymbol{\delta}\right]$-TORSION FREE CONTROLLABILITY}

In this section we establish two criteria for $k\left[\frac{d}{d t}, \delta\right]$-free and $k\left[\frac{d}{d t}, \delta\right]$ torsion free controllability. The first one uses the resolution of Serre's conjecture [44] due to Quillen [40] and Suslin [47] (see also [50] and [26] for a detailed exposition).

In the present context, Quillen-Suslin's theorem may be stated as:

Proposition 3.1. A delay system is $k\left[\frac{d}{d t}, \delta\right]$-free controllable if, and only if, it is $k\left[\frac{d}{d t}, \delta\right]$-projective controllable.

This proposition implies the following criterion:

Theorem 3.2. A delay system $\Lambda$ with presentation matrix $P_{\Lambda}$ of full generic rank $\beta$ is $k\left[\frac{d}{d t}, \delta\right]$-free controllable if, and only if,

$$
\forall\left(s, z_{1}, \ldots, z_{r}\right) \in \bar{k}^{r+1}, \quad r k_{\bar{k}} P_{\Lambda}\left(s, z_{1}, \ldots, z_{r}\right)=\beta
$$

where $\bar{k}$ is the algebraic closure of $k$. This rank criterion is equivalent to the common minors of $P_{\Lambda}$ of order $\beta$ having no common zero in $\bar{k}^{r+1}$.

Proof. Proposition 20.8, p. 495, from Eisenbud [9] (see also Buchsbaum and Eisenbud [6]) establishes the equivalence between projectivity of $\Lambda$ and equality to $k\left[\frac{d}{d t}, \delta\right]$ of the ideal $\mathfrak{I}$ generated by the $\beta^{\text {th }}$ order minors of $P_{\Lambda}\left(\frac{d}{d t}, \delta\right)$. Hilbert's Nullstellensatz implies that the equality $\mathfrak{I}=k\left[\frac{d}{d t}, \boldsymbol{\delta}\right]$ is equivalent to these minors having no common zero in $\bar{k}^{r+1}$, i.e., no loss of rank for $P_{\Lambda}\left(s, z_{1}, \ldots, z_{r}\right)$ for all $\left(s, z_{1}, \ldots, z_{r}\right)$ in $\bar{k}^{r+1}$.

A criterion for $k\left[\frac{d}{d t}, \delta\right]$-torsion free controllability is the following:

Theorem 3.3. A delay system $\Lambda$ is $k\left[\frac{d}{d t}, \delta\right]$-torsion free controllable if, and only if, the gcd of the $\beta \times \beta$ minors of $P_{\Lambda}$ belongs to $k$.

Proof. This criterion readily follows from Theorem 2 in Youla and Gnavi [49]. According to the latter, the gcd of the $\beta \times \beta$ minors of $P_{\Lambda}$ belongs to $k$ if, and only if, for all $i$ in $\{0, \ldots, r\}$, there exists matrices $Q_{i}$, with coefficients in $k\left[\frac{d}{d t}, \boldsymbol{\delta}\right]$, such that:

$$
P_{\Lambda} Q_{i}=\psi_{i} I_{\beta}
$$

where $\psi_{i}$ is a polynomial in $k\left[\frac{d}{d t}, \delta\right]$ which does not depend on the variable with index $i$ ( $\frac{d}{d t}$ having index $i=0$ and $\delta_{i}$ index $i$ for $i \geqslant 1$ ). The equation $P_{\Lambda} Q_{0}=\psi_{0} I_{\beta}$ expresses the freeness of

$$
\Lambda_{0}=k\left(\delta_{1}, \ldots, \delta_{r}\right)\left[\frac{d}{d t}\right] \otimes_{k[d / d t, \boldsymbol{\delta}]} \Lambda
$$

the equation $P_{\Lambda} Q_{1}=\psi_{1} I_{\beta}$, the freeness of

$$
\Lambda_{1}=k\left(\frac{d}{d t}, \delta_{2}, \ldots, \delta_{r}\right)\left[\delta_{1}\right] \otimes_{k[d / d t, \boldsymbol{\delta}]} \Lambda, \ldots
$$


and $P_{\Lambda} Q_{r}=\psi_{r} I_{\beta}$ expresses the freeness of

$$
\Lambda_{r}=k\left(\frac{d}{d t}, \delta_{1}, \ldots, \delta_{r-1}\right)\left[\delta_{r}\right] \otimes_{k[d / d t, \boldsymbol{\delta}]} \Lambda .
$$

All the mentioned rings being principal, the freeness of the $\Lambda_{i}$ is equivalent to their torsion freeness. Evidently, $\Lambda$ is torsion free if, and only if, each of the $\Lambda_{i}$ 's is so.

\section{Relationships With VARIOUS COPRIMENESS NOTIONS}

Let us indicate some relationships between the above algebraic controllablity notions and some generalizations of the matrix coprimeness over $k\left[\frac{d}{d t}\right]$. Consider for example a dynamics $\mathcal{D}$ over $k\left[\frac{d}{d t}, \boldsymbol{\delta}\right]$ with input $\boldsymbol{u}$ and output $\boldsymbol{y}$ and with equations:

$$
A\left(\frac{d}{d t}, \boldsymbol{\delta}\right) \boldsymbol{y}=B\left(\frac{d}{d t}, \boldsymbol{\delta}\right) \boldsymbol{u}
$$

Different types of coprimeness have been defined in, e.g., [49], [18]. The matrices $A$ and $B$ are said to be zero prime (resp. minor prime, variety prime) if the maximal order minors of $P_{\mathcal{D}}$ have no common zero in $\bar{k}^{r+1}$ (resp. have gcd 1 in $k\left[\frac{d}{d t}, \delta\right]$, generate an ideal containing polynomials of each of the rings $k\left[\frac{d}{d t}\right], k\left[\delta_{1}\right], \ldots$, and $\left.k\left[\delta_{r}\right]\right)$. They are called factor prime, if for any decomposition $P_{\mathcal{D}}=P_{1} P_{2}$ in $k\left[\frac{d}{d t}, \delta\right]$ with $P_{1}$ square, $P_{1}$ is unimodular.

We have the following direct Corollaries of Theorems 3.2 and 3.3.

Corollary 4.1. The matrices $A$ and $B$ of equation (4.1) are zero prime (resp. minor prime) if, and only if, $\mathcal{D}$ is $k\left[\frac{d}{d t}, \delta\right]$-free controllable (resp. $k\left[\frac{d}{d t}, \delta\right]$-torsion free controllable).

Corollary 4.2. The matrices $A$ and $B$ of equation (4.1) are variety prime if, and only if, $\mathcal{D}$ is free controllable simultaneously on $k\left(\delta_{1}, \ldots, \delta_{r}\right)\left[\frac{d}{d t}\right]$, $k\left(\frac{d}{d t}, \delta_{2}, \ldots, \delta_{r}\right)\left[\delta_{1}\right], \ldots$, and $k\left(\frac{d}{d t}, \delta_{1}, \ldots, \delta_{r-1}\right)\left[\delta_{r}\right]$.

In the case of a single delay, the notions of factor primeness and minor primeness coincide (see [49, Theorem 1$]$ ). The case of several delays is more complex. The matrices $A$ and $B$ may indeed be factor prime with a non torsion free underlying module. The following example is given in [49]; let $\mathcal{D}=\left[u_{1}, u_{2}, y_{1}, y_{2}\right]$ be the dynamics with equations:

$$
\begin{aligned}
\left(\delta_{1} \delta_{2}-\frac{d^{3}}{d t^{3}}\right) y_{1}+\left(\delta_{1}^{2}-\frac{d}{d t} \delta_{2}\right) y_{2} & =\frac{d}{d t} u_{1} \\
\left(\delta_{2}^{2}-\frac{d^{2}}{d t^{2}} \delta_{1}\right) y_{1}+\left(\delta_{1} \delta_{2}-\frac{d^{3}}{d t^{3}}\right) y_{2} & =\frac{d}{d t} u_{2} .
\end{aligned}
$$

The corresponding matrices $A$ and $B$ of $\mathcal{D}$ are factor prime. Additionally, multiplying the first equation by $\delta_{2}$ and the second by $-\delta_{1}$, we obtain:

$$
\frac{d}{d t}\left[\delta_{1}^{2} \dot{y}_{1}-\delta_{2} \ddot{y}_{1}+\delta_{1} \ddot{y}_{2}-\delta_{2}^{2} y_{2}-\delta_{2} u_{1}+\delta_{1} u_{2}\right]=0
$$

wherefrom a torsion element.

ESAIM: Cocv, SEPTEMber 1998, Vol. 3, 301-314 


\section{EXAMPLE: $\pi$-FREENESS AND TRANSMISSION DELAY}

Suppose we have two $k\left[\frac{d}{d t}\right]$-systems $\Lambda_{1}$ and $\Lambda_{2}$ in cascade with a transmission delay operator $\pi \in k[\delta], \pi \notin k$, between them. Denote this cascade by $\Lambda_{1} \star \pi \star \Lambda_{2}$ and by $\Lambda_{1} \star \Lambda_{2}$ the two systems in series without any transmission delay between them $\left(\Lambda_{1} \star \Lambda_{2}\right.$ is thus a $k\left[\frac{d}{d t}\right]$-system $)$. For the sake

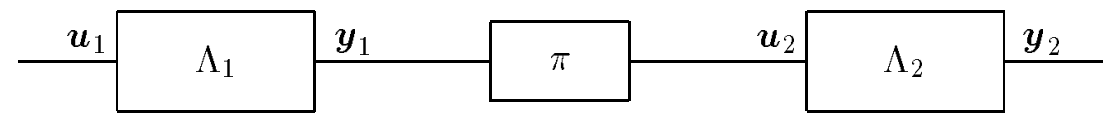

Figure 1 . The system $\Lambda_{1} \star \pi \star \Lambda_{2}$

of simplicity, take two $k\left[\frac{d}{d t}\right]-$ systems with equations

$$
A_{i}\left(\frac{d}{d t}\right) \boldsymbol{y}_{i}=B_{i}\left(\frac{d}{d t}\right) \boldsymbol{u}_{i}
$$

where $\boldsymbol{u}_{i}$ and $\boldsymbol{y}_{i}$ have $m$ elements, $A_{i}, B_{i} \in k\left[\frac{d}{d t}\right]^{m \times m}, i=1$, 2, and

$$
\boldsymbol{u}_{2}=\pi \boldsymbol{y}_{1} \text {. }
$$

Set $\boldsymbol{u}=\boldsymbol{u}_{1}, \boldsymbol{y}=\boldsymbol{y}_{2}$ and $\Lambda_{1} \star \pi \star \Lambda_{2}=\left[\boldsymbol{u}, \boldsymbol{y}_{1}, \boldsymbol{y}\right]$ with equations (5.1). We have the following result, which could be seen as a complement to [14]:

Proposition 5.1. The $k\left[\frac{d}{d t}\right]$-system $\Lambda_{1} \star \Lambda_{2}$ is controllable if, and only if, the delay system $\Lambda_{1} \star \pi \star \Lambda_{2}$ is $\pi$-free.

Proof. The "if" part is obvious. For the "only if" part, take a basis $\boldsymbol{b}=$ $\left(b_{1}, \ldots, b_{m}\right)$ of the $k\left[\frac{d}{d t}\right]-\bmod u l e \Lambda_{1} \star \Lambda_{2}$. Then $1 \otimes \boldsymbol{b}$ is also a basis of the free $k\left[\frac{d}{d t}, \pi, \pi^{-1}\right]$-module $k\left[\frac{d}{d t}, \pi, \pi^{-1}\right] \otimes_{k[d / d t]} \Lambda_{1} \star \Lambda_{2}$, and any system variable of $\Lambda_{1}\left(\operatorname{resp} . \Lambda_{2}\right)$ is a $k\left[\frac{d}{d t}\right]$-linear combination of $1 \otimes \boldsymbol{b}$ (resp. $\pi^{-1}(1 \otimes \boldsymbol{b})$ ).

The following peculiar case of proposition 5.1 is stated in a more classical fashion.

Corollary 5.2. Consider the $k\left[\frac{d}{d t}, \boldsymbol{\delta}\right]$-dynamics $\Gamma=[\boldsymbol{x}, \boldsymbol{u}]$ with equations

$$
\dot{\boldsymbol{x}}=F \boldsymbol{x}+G \pi \boldsymbol{u}
$$

where $F \in k^{n \times n}, G \in k^{n \times m}, \pi \in k[\delta], \pi \notin k$, and the $k\left[\frac{d}{d t}\right]$-dynamics $\widetilde{\Gamma}=[\tilde{\boldsymbol{x}}, \tilde{\boldsymbol{u}}]$ with equations

$$
\dot{\tilde{\boldsymbol{x}}}=F \tilde{\boldsymbol{x}}+G \tilde{\boldsymbol{u}} .
$$

The following two properties are equivalent:

(i) The $k\left[\frac{d}{d t}, \delta\right]$-dynamics $\Gamma$ is $\pi$-free;

(ii) The $k\left[\frac{d}{d t}\right]$-dynamics $\widetilde{\Gamma}$ is controllable in Kalman's sense, i.e., $r k_{k}\left[G, F G, \ldots, F^{n-1} G\right]=n$.

REMARK 5.3. In most practical case studies, $\pi$ is a simple delay operator $\delta$. However, this may not be the case, e.g., for delay systems steming from wave equations with appropriate boundary conditions. See $[32,37]$ for examples of long electric lines. 


\section{Reachability - WEAK CONTROLlability}

Consider the $k\left[\frac{d}{d t}, \boldsymbol{\delta}\right]$-dynamics $\Gamma=[\boldsymbol{x}, \boldsymbol{u}]$ with equations

$$
\dot{\boldsymbol{x}}=F(\boldsymbol{\delta}) \boldsymbol{x}+G(\boldsymbol{\delta}) \boldsymbol{u}
$$

where $\boldsymbol{x}=\left(x_{1}, \ldots, x_{n}\right), \boldsymbol{u}=\left(u_{1}, \ldots, u_{m}\right)$, and the matrices $F(\boldsymbol{\delta}) \in k[\boldsymbol{\delta}]^{n \times n}$ and $G(\delta) \in k[\delta]^{n \times m}$.

Criteria for the reachability (see, e.g., [5], [45]) and the weak controllability (see [31]) of $\Gamma$ are:

- The dynamics $\Gamma$ with equations (6.1) is reachable if, and only if,

$$
\forall\left(s, z_{1}, \ldots, z_{r}\right) \in \bar{k}^{r+1}, \quad \operatorname{rk}_{\bar{k}}\left[s I_{n}-F\left(z_{1}, \ldots, z_{r}\right) \mid G\left(z_{1}, \ldots, z_{r}\right)\right]=n .
$$

- It is weakly controllable if, and only if,

$$
\operatorname{rk}_{k(\boldsymbol{\delta})}\left[G(\boldsymbol{\delta}), \ldots, F(\boldsymbol{\delta})^{n-1} G(\boldsymbol{\delta})\right]=n .
$$

The following propositions give an interpretation of the preceding notions in our setting.

Proposition 6.1. The dynamics $\Gamma$ is reachable if, and only if, $\Gamma$ is free controllable over $k\left[\frac{d}{d t}, \delta\right]$.

Proof. Follows directly from Theorem 3.2.

Proposition 6.2. The dynamics $\Gamma$ is weakly controllable if, and only if, $\Gamma$ is free controllable over $k(\boldsymbol{\delta})\left[\frac{d}{d t}\right]$.

Proof. Taking $k(\boldsymbol{\delta})\left[\frac{d}{d t}\right] \otimes_{k[d / d t, \boldsymbol{\delta}]} \Gamma$ amounts, with $K=k(\boldsymbol{\delta})$, to considering the $K\left[\frac{d}{d t}\right]$-module $\Gamma$ as a $K\left[\frac{d}{d t}\right]$-system. One is then in the framework of [10] and the controllability is expressed either as $k(\boldsymbol{\delta})\left[\frac{d}{d t}\right] \otimes_{k[d / d t, \boldsymbol{\delta}]} \Gamma$ free or as $\operatorname{rk}_{k(\boldsymbol{\delta})}\left[G(\boldsymbol{\delta}), \ldots, F(\boldsymbol{\delta})^{n-1} G(\boldsymbol{\delta})\right]=n$.

\section{Controllability interpretations AND COMParison DIAGRAM}

Before giving a diagram on which most controllability notions can be compared, let us recall the interpretations of two notions defined in a functional analytic framework (see $[32,33]$ for details). In this section, $k=\mathbb{R}$ or $\mathbb{C}$.

\subsection{Spectral controllability}

In this and the following section, we use the ring $k\left[s, e^{-\boldsymbol{h} s}\right]$, viewed as a subring of the convergent power series ring $k\{\{s\}\}$ (where $s$ plays the role of $\frac{d}{d t}$, and $\boldsymbol{h}=\left(h_{1}, \ldots, h_{r}\right)$, the $h_{i}$ 's $\left(h_{i} \in \mathbb{R}, h_{i}>0\right)$ being the amplitudes of the corresponding delays). As previously noted [24], [46], mapping $\frac{d}{d t} \mapsto s$, $\delta_{i} \mapsto e^{-h_{i} s}$ yields an isomorphism between the rings $k\left[\frac{d}{d t}, \delta\right]$ and $k\left[s, e^{-\boldsymbol{h} s}\right]$, where $e^{ \pm \boldsymbol{h} s}=\left(e^{ \pm h_{1} s}, \ldots, e^{ \pm h_{r} s}\right)$. Thus, by a slight abuse of language, a finitely generated $k\left[s, e^{-\boldsymbol{h} s}\right]$-module will still be called a delay system.

The following definition of spectral controllability extends previous ones (see, e.g., [3], [38], [1], [41]) in our context.

ESAIM: CoCv, September 1998, Vol. 3, 301-314 
Definition 7.1. Let $\Lambda$ be a delay system defined over the ring $k\left[s, e^{-\boldsymbol{h} s}\right]$, with presentation matrix $P_{\Lambda}$ of full generic rank $\beta$. It is called spectrally controllable if

$$
\forall s \in \mathbb{C}, \quad \operatorname{rk}_{\mathbb{C}} P_{\Lambda}\left(s, e^{-\boldsymbol{h} s}\right)=\beta .
$$

Set $\mathfrak{S}_{r}=k(s)\left[e^{-\boldsymbol{h} s}, e^{\boldsymbol{h} s}\right] \cap \mathfrak{E}$, where $\mathfrak{E}$ denotes the ring of entire functions. We have the following interpretation of spectral controllability:

Proposition 7.2. Let $\Lambda$ be a delay system over $k\left[s, e^{-h s}\right]$, such that $\Lambda$ is $k\left[s, e^{-\boldsymbol{h} s}, e^{\boldsymbol{h} s}\right]$-torsion free controllable. Then $\Lambda$ is spectrally controllable if, and only if, it is $\mathfrak{S}_{r}$-torsion free controllable.

See [19] for related results.

\subsection{Approximate controllability}

The approximate controllability corresponds to the density of the reachable space in a given function space (see, e.g. $[1,38]$ ). For a $k\left[\frac{d}{d t}, \delta\right]$ dynamics $\mathcal{D}=[\boldsymbol{u}, \boldsymbol{x}]$ with equations

$$
\dot{\boldsymbol{x}}(t)=\sum_{i=1}^{N} F_{-i} \dot{\boldsymbol{x}}(t-i h)+\sum_{i=0}^{N} F_{i} \boldsymbol{x}(t-i h)+\sum_{j=0}^{M} G_{j} \boldsymbol{u}(t-j h)
$$

where $\boldsymbol{x}(t) \in k^{n}, \boldsymbol{u}(t) \in k^{m}, h \in \mathbb{R}_{+}, h>0, M \leqslant N, F_{-i}, F_{i} \in k^{n \times n}$, and $G_{j} \in k^{n \times m}$, a matrix criterion (see [1], [48]) is given by:

$$
\begin{array}{ll}
\operatorname{rk}_{\mathbb{C}}[\mathcal{F}(s) \mid \mathcal{G}(s)]=n, & \text { for all } s \in \mathbb{C}, \quad \text { and } \\
\operatorname{rk}_{\mathbb{C}}\left[s F_{-N}+F_{N} \mid G_{M}\right]=n, & \text { for almost all } s \in \mathbb{C} .
\end{array}
$$

with

$$
\begin{aligned}
\mathcal{F}(s) & =s I-F_{-1} s e^{-s}-\cdots-F_{-N} s e^{-N s}-F_{0}-F_{1} e^{-s}-\cdots-F_{N} e^{-N s} \\
\mathcal{G}(s) & =F_{0}+F_{1} e^{-s}-\cdots-G_{-M} e^{-M s} .
\end{aligned}
$$

Consider the dynamics $\widetilde{\mathcal{D}}=k\left[s, e^{-h s}, e^{h s}\right] \otimes_{k\left[s, e^{-h s}\right]} \mathcal{D}$. Let $\delta_{h_{i}}$ and $\delta_{a_{i}}$ be the delays of respective amplitudes $h_{i}$ and $a_{i}$. Let also $\boldsymbol{v}=e^{(N-M) h s} \boldsymbol{u}$ be a new input of $\widetilde{\mathcal{D}}$. Then:

Proposition 7.3. The dynamics $\mathcal{D}$ is approximately controllable if, and only if, the two following conditions are satisfied:

(i) it is spectrally controllable,

(ii) the $k\left[s, e^{-h s}, e^{h s}\right]$-module $\delta^{-N} \widetilde{\mathcal{D}} / \delta^{-1} \widetilde{\mathcal{D}}$ is torsion free.

\subsection{THE COMPARISON DIAGRAM}

Consider the single delay dynamics $\mathcal{D}=[\boldsymbol{u}, \boldsymbol{x}]$ with equations

$$
\dot{\boldsymbol{x}}=F(\delta) \boldsymbol{x}+G(\delta) \boldsymbol{u}
$$

where $F$ and $G$ are matrices of appropriate sizes. Setting

$$
\begin{aligned}
\langle F(\delta), G(\delta)\rangle & =\left[G(\delta), \ldots, F(\delta)^{n-1} G(\delta)\right] \\
\Delta(s, z) & =[s I-F(z) \mid G(z)]
\end{aligned}
$$

yields the implications and equivalences diagram depicted in Figure 2. The following result gives implication relationships between the new notion of 


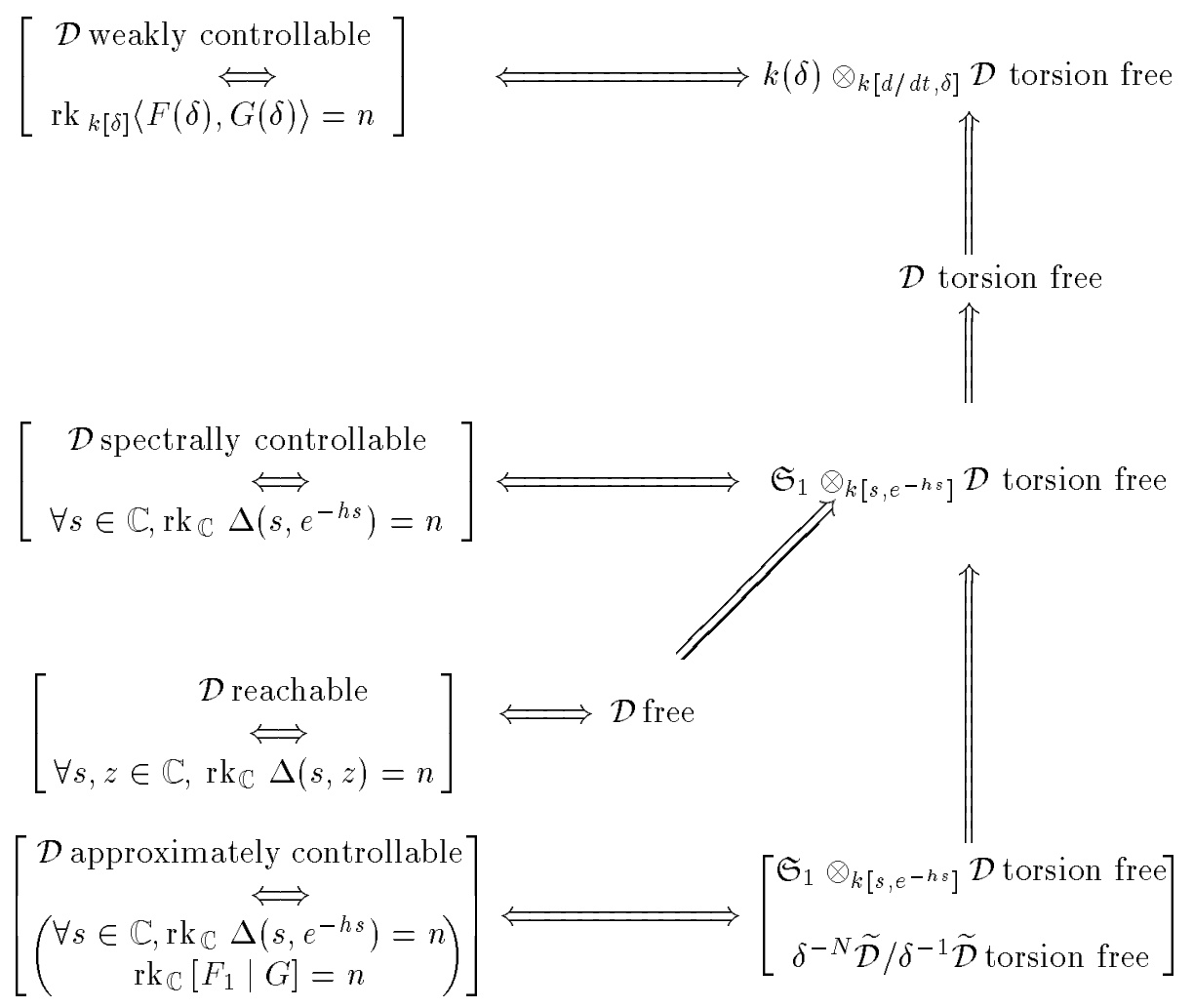

Figure 2. Controllability implications and equivalences diagram

$\delta_{1}^{\alpha_{1}} \ldots \delta_{r}^{\alpha_{r}}$-freeness, where $\alpha_{1}, \ldots, \alpha_{r}$ are non-negative integers, $\alpha_{1}+\ldots+$ $\alpha_{r}>0$, and the ones in the diagram of Figure 2.

Proposition 7.4. Let $\Lambda$ be a $k\left[\frac{d}{d t}, \delta\right]$-system. The following chain of implications is true

$\Lambda$ spectrally controllable $\Longrightarrow \Lambda \delta_{1}^{\alpha_{1}} \ldots \delta_{r}^{\alpha_{r}}$ free $\Longrightarrow \Lambda k\left[\frac{d}{d t}, \delta\right]$-torsion free

Proof. The proof follows directly from the inclusion chain

$$
k\left[\frac{d}{d t}, \boldsymbol{\delta}\right] \subset k\left[\frac{d}{d t}, \delta, \delta^{-1}\right] \subset \mathfrak{S}_{r} .
$$

\section{INTERPRETATIONS OF OBSERVABILITY NOTIONS}

Consider the single delay dynamics $\mathcal{D}=[\boldsymbol{u}, \boldsymbol{y}]$ with equations

$$
\begin{aligned}
& \dot{\boldsymbol{x}}=F(\delta) \boldsymbol{x}+G(\delta) \boldsymbol{u} \\
& \boldsymbol{y}=H(\delta) \boldsymbol{x}
\end{aligned}
$$


where $\boldsymbol{x}=\left(x_{1}, \ldots, x_{n}\right), \boldsymbol{u}=\left(u_{1}, \ldots, u_{m}\right), \boldsymbol{y}=\left(y_{1}, \ldots, y_{p}\right), F(\delta) \in k[\delta]^{n \times n}$, $G(\delta) \in k[\delta]^{n \times m}$, and $H(\delta) \in k[\delta]^{p \times n}$. Set:

$$
\mathcal{O}(\delta)=\left[\begin{array}{c}
H(\delta) \\
H(\delta) F(\delta) \\
\vdots \\
H(\delta) F^{n-1}(\delta)
\end{array}\right]
$$

The following observability notions are taken from [29]. The dynamics $\mathcal{D}$ is said observable over $k[\delta]$ (resp. over $k(\delta)$ ) (in the sense of [29]) if $\mathcal{O}(\delta)$ has a left inverse in $k[\delta]^{n \times(n p)}$ (resp. in $k(\delta)^{n \times(n p)}$ ). It is said hyperobservable if the kernel of $\mathcal{O}$, considered as an operator defined over the ring of continuous functions $C^{0}\left[k, k^{n}\right]$ is trivial. It is spectrally observable if $\mathrm{rk} \mathbb{C}\left(e^{-\boldsymbol{h} s}\right)=n$ for all $s \in \mathbb{C}$.

Setting

$$
\Omega(s, z)=\left[\begin{array}{c}
s I_{n}-F(z) \\
H(z)
\end{array}\right]
$$

we have the following interpretations:

Proposition 8.1. The $k\left[\frac{d}{d t}, \delta\right]$-dynamics $\mathcal{D}$ is observable over $k[\delta]$ (resp. over $k(\delta)$ ) in the sense of [29] if, and only if, it is $k\left[\frac{d}{d t}, \delta\right]$-observable (resp. $k(\delta)\left[\frac{d}{d t}\right]-$ observable $)$.

Proof. It is shown in [29] that observability over $k[\delta]$ of $\mathcal{D}$ is equivalent to:

$$
\forall(s, z) \in \bar{k}^{2}, \quad \operatorname{rk}_{\bar{k}} \Omega(s, z)=n
$$

which is in turn equivalent to the left invertibility of $\Omega\left(\frac{d}{d t}, \delta\right)$ over $k\left[\frac{d}{d t}, \delta\right]$. Additionally, the relations of $\mathcal{D}$ being

$$
\Omega\left(\frac{d}{d t}, \delta\right) \boldsymbol{x}-\left[\begin{array}{cc}
G(\delta) & 0 \\
0 & -I_{p}
\end{array}\right]\left[\begin{array}{l}
\boldsymbol{u} \\
\boldsymbol{y}
\end{array}\right]=0
$$

the property (8.3) is obviously equivalent to the $k\left[\frac{d}{d t}, \delta\right]$-observability of $\mathcal{D}$.

The proof of the observability over $k(\delta)$ is analogous.

Proposition 8.2. The $k\left[\frac{d}{d t}, \delta\right]$-dynamics $\mathcal{D}$ is observable if, and only if, it is $k\left[\frac{d}{d t}, \delta, \delta^{-1}\right]$-observable.

Proof. It is shown in [29] that hyperobservability of $\mathcal{D}$ is equivalent to:

$$
\forall(s, z) \in \bar{k}^{2}, z \neq 0, \quad \operatorname{rk}_{\bar{k}} \Omega(s, z)=n .
$$

This criterion is equivalent to the projectivity of

$$
k\left[\frac{d}{d t}, \delta, \delta^{-1}\right] \otimes_{k[d / d t, \delta]}(\mathcal{D} /[\boldsymbol{u}, \boldsymbol{y}])
$$

this module being given by its generators $[\overline{\boldsymbol{x}}]$ and its relations

$$
\Omega\left(\frac{d}{d t}, \delta\right) \bar{x}=\overline{0} .
$$

The presentation matrix of this module being left invertible in $k\left[\frac{d}{d t}, \delta, \delta^{-1}\right]$, $\overline{\boldsymbol{x}}=\overline{\mathbf{0}}$, which implies $\boldsymbol{x} \in k\left[\frac{d}{d t}, \delta, \delta^{-1}\right] \otimes_{k[d / d t, \delta]}[\boldsymbol{u}, \boldsymbol{y}]$. 


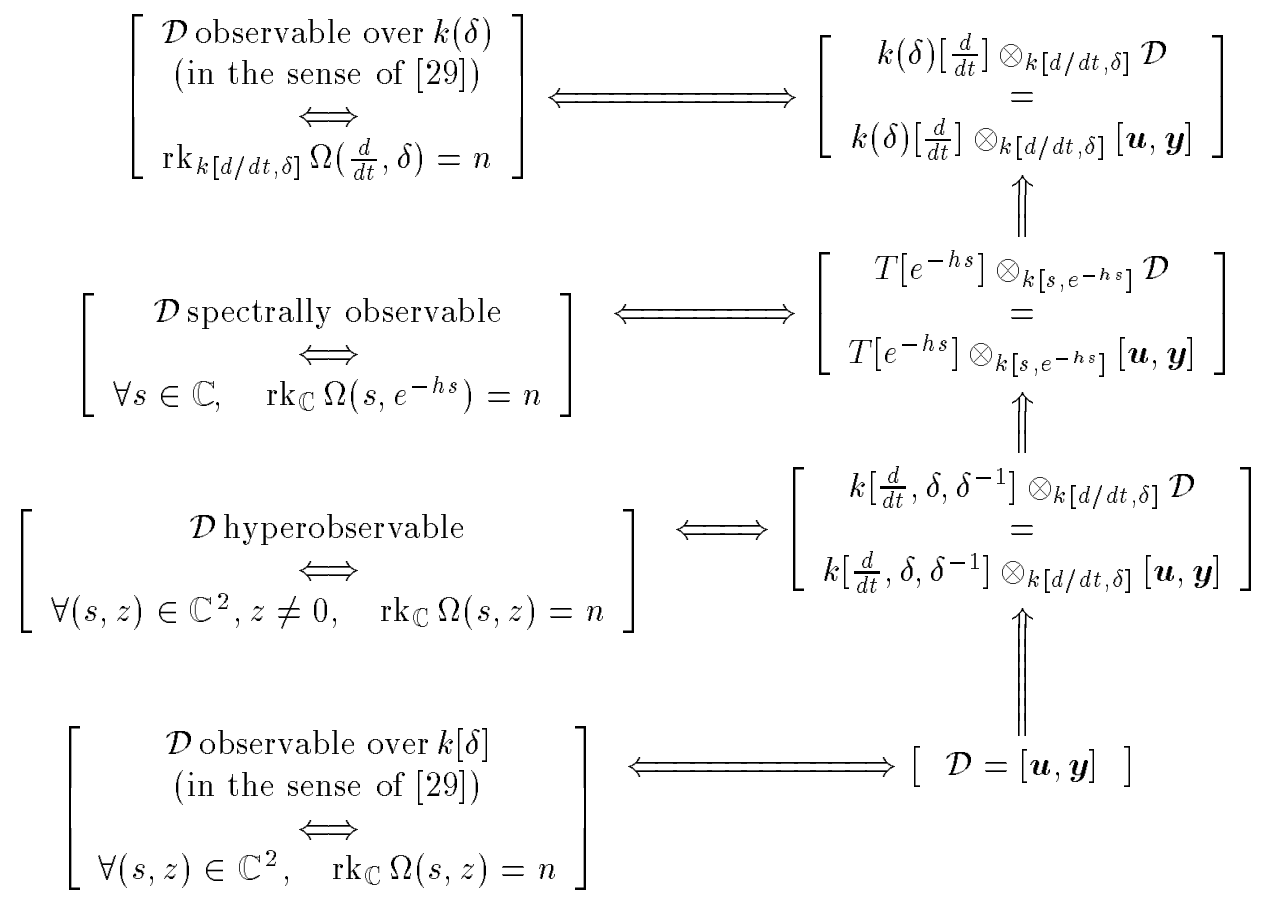

FiguRE 3. Observability implications and equivalences diagram

Let $T\left[e^{-h s}\right]$ be the polynomial ring in $e^{-h s}$ with coefficients in $T=$ $k\left(s, e^{-h s}\right) \cap T_{\mathbb{C}}$, where $T_{\mathbb{C}}$ is the subring of $\mathbb{C}\left(s, e^{-h s}\right)$ generated by the family

$$
\left\{b \frac{d^{i} \theta_{\alpha}}{d s^{i}} \mid b \in \mathbb{R}, \alpha \in \mathbb{C}, i \in \mathbb{N}\right\}
$$

and $\theta_{\alpha}$ designates the entire function

$$
\frac{1-e^{-h(s-\alpha)}}{s-\alpha} .
$$

We then have

Proposition 8.3. The dynamics $\mathcal{D}$ is spectrally observable if, and only if, it is $T\left[e^{-h s}\right]-$ observable.

Proof. It is shown in [25] that spectral observability of $\mathcal{D}$ is equivalent to the left invertibility of $\Omega\left(s, e^{-h s}\right)$ over $T\left[e^{-h s}\right]$, which readily implies the $T\left[e^{-h s}\right]$-observability.

The implications and equivalences diagram for the various observability notions of the dynamics $\mathcal{D}$ is depicted in Figure 3. 


\section{REFERENCES}

[1] Z. Bartosiewicz: Approximate controllability of neutral systems with delays in control, J. Diff. Eq., 51, 1984, 295-325.

[2] A. Bensoussan, G. Da Prato, M.C. Delfour, S.K. Mitter: Representation and Control of Infinite Dimensional Systems, 1, 2, Birkhäuser, Boston, 1992-1993.

[3] K.P.M. Bhat, H.N. Koivo: Modal characterizations of controllability and observability for time-delay systems, IEEE Trans. Automat. Contr., 21, 1976, 292-293.

[4] H. Bourlès, M. Fliess: Finite poles and zeros of linear systems: an intrinsic approach, Internat J. Control, 68, 1997, 897-922.

[5] J.W. Brewer, J.W. Bunce, F.S. Van Vleck: Linear Systems over Commutative Rings, Marcel Dekker, New York, 1986.

[6] D.A. Buchsbaum, D. Eisenbud: What makes a complex exact? J. Alg., 25, 1973, $259-268$.

[7] C.I. Byrnes: On the control of certain deterministic, infinite-dimensional systems by algebro-geometric techniques, Amer. J. Math., 100, 1978, 1333-1381.

[8] R.M. Cohn: A difference-differential basis theorem, Canad. J. Math., 22, 1970, $1224-$ 1237.

[9] D. Eisenbud: Commutative Algebra with a View toward Algebraic Geometry, SpringerVerlag, New York, 1995.

[10] M. Fliess: Some basic structural properties of generalized linear systems, Systems Control Lett., 15, 1990, 391-396.

[11] M. Fliess: A remark on Willems' trajectory characterization of linear controllability, Systems Control Lett., 19, 1992, 43-45.

[12] M. Fliess: Reversible linear and non linear discrete time dynamics, IEEE Trans. Automat. Contr., 37, 1992, 1144-1153.

[13] M. Fliess: Une interprétation algébrique de la transformation de Laplace et des matrices de transfert, Linear Alg. Appl., 203-204, 1994, 429-442.

[14] M. Fliess, H. Bourlès: Discussing some examples of linear systems interconnections, Systems Control Lett, 27, 1996, 1-7.

[15] M. Fliess, R. Hotzel: Sur les systèmes linéaires à dérivation non entière, C.R. Acad. Sci. Paris II, 324, 1997, 99-105.

[16] M. Fliess, H. Mounier: Quelques propriétés structurelles des systèmes linéaires à retards constants, C. R. Acad. Sci. Paris I, 319, 1994, 289-294.

[17] M. Fliess, H. Mounier: Interpretation and comparison of various types of delay system controllabilities, In Proc. IFAC Conference System, Structure and Control, Nantes, 1995, 330-335.

[18] E. Fornasini, M.E. Valcher: A polynomial matrix approach to the behavioral analysis of $n d$ systems, In 3rd European Control Conference Proc., Rome, 1995, 1757-1762.

[19] H. Glüsing-Lüerßen: A behavioral approach to delay differential systems, SIAM J. Contr. Opt., 35, 1997, 480-499.

[20] A. Grothendieck, J.A. Dieudonné: Eléments de géométrie algébrique I, SpringerVerlag, Berlin, 1971.

[21] R. Hartshorne: Algebraic Geometry, Springer-Verlag, New York, 1977.

[22] R.E. Kalman, P.L. Falb, M.A. Arbib: Topics in Mathematical Systems Theory, McGraw-Hill, New York, 1969.

[23] E.W. Kamen: On an algebraic theory of systems defined by convolution operators, Math. Syst. Theory, 9, 1975, 57-74.

[24] E.W. Kamen: An operator theory of linear functional differential equations, J. Diff. Eq. 27, 1978, 274-297.

[25] E.W. Kamen, P.P. Khargonekar, A. Tannenbaum: Proper stable Bezout factorization and feedback control of linear time-delay systems, Internat. J. Control, 43, 1986, 837857.

[26] T.Y. Lam: Serre's Conjecture, Springer-Verlag, Berlin, 1978.

[27] S. Lang: Algebra, $3^{\text {rd }}$ ed., Addison-Wesley, Reading, MA, 1993.

[28] E.B. Lee, S. Neftci, A. Olbrot: Canonical forms for time delay systems, IEEE Trans. Automat. Contr., 27, 1982, 128-132. 
[29] E.B. Lee, A. Olbrot: Observability and related structural results for linear hereditary systems, Internat. J. Control, 34, 1981, 1061-1078.

[30] A. Manitius, R. Triggiani: Function space controllability of retarded systems: a derivation from abstract operator conditions, SIAM J. Contr. Opt., 16, 1978, 599645.

[31] A.S. Morse: Ring models for delay-differential systems, Automatica, 12, 1976, 529531.

[32] H. Mounier: Propriétés structurelles des systèmes linéaires à retards : aspects théoriques et pratiques, Thèse, Université Paris-Sud, Orsay, 1995.

[33] H. Mounier: Algebraic interpretations of the spectral controllability of a linear delay system, Forum Math., 10, 1998, 39-58.

[34] H. Mounier: Stabilization of a class of linear delay systems, Math. Comp. Sim., 45, 1998, 329-338.

[35] H. Mounier, J. Rudolph, M. Fliess, P. Rouchon: Tracking control of a vibrating string with an interior mass viewed as a delay system, ESAIM: Control Optimisation and Calculus of Variations, http://www. emath.fr/cocv/, 3, 1998, 315-321.

[36] H. Mounier, P. Rouchon, J. Rudolph: Some examples of linear delay systems, RAIROJESA-APII, 31, 1997, 911-925.

[37] H. Mounier, P. Rouchon, J. Rudolph: $\pi$-freeness of a long electric line, Comput. Eng. in Syst. Appl. IMACS Multiconference, Lille, 1996, 28-29.

[38] D.A. O'Connor, T.J. Tarn: On the function space controllability of linear neutral systems, SIAM J. Contr. Opt., 21, 1983, 306-329.

[39] P. Picard, J.F. Lafay: Further results on controllability of linear systems with delays, In European Control Conference Proc., Rome, 1995, 3313-3318.

[40] D. Quillen: Projective modules over polynomial rings, Inv. Math., 36, 1976, 167-171.

[41] P. Rocha, J. Willems: Behavioral controllability of D-D systems, SIAM J. Contr. Opt., 35, 1997, 254-264.

[42] J. Rotman: An Introduction to Homological Algebra, Academic Press, New-York, 1979.

[43] L.H. Rowen: Ring Theory, Academic Press, Boston, 1991.

[44] J.P. Serre: Faisceaux algébriques cohérents, Annals of Math., 61, 1955, 197-278.

[45] E.D. Sontag: Linear systems over commutative rings: a survey, Richerche di Automatica, 7, 1976, 1-34.

[46] M.W. Spong, T.J. Tarn: On the spectral controllability of delay-differential equations, IEEE Trans. Automat. Contr., 26, 1981, 527-528.

[47] A.A. Suslin: Projective modules over a polynomial ring are free (in Russian), Dokl. Akad. Nauk. S.S.S.R., 229, 1976, 1063-1066; English translation: Soviet Math. Dokl., 17, 1160-1164.

[48] Y. Yamamoto: Reachability of a class of infinite-dimensional linear systems: an external approach with applications to general neutral systems, SIAM J. Contr. Opt., 27, 1989, 217-234.

[49] D.C. Youla, G. Gnavi: Notes on n-dimensional system theory, IEEE Trans. Circuits Syst., 26, 1979, 105-111.

[50] D.C. Youla, P.F. Pickel: The Quillen-Suslin theorem and the structure of ndimensional elementary polynomial matrices, IEEE Trans. Circuits Syst., 31, 1984, 513-518. 